\title{
ONE-PARAMETER SEMIGROUPS IN A SEMIGROUP( $\left.{ }^{(}\right)$
}

BY

\author{
PAUL S. MOSTERT AND ALLEN L. SHIELDS
}

1. Introduction. A topological semigroup is a Hausdorff space $S$ with a continuous, associative multiplication. If there is an identity, 1, then $H(1)$ will denote the maximal subgroup of $S$ containing 1 ; in other words, $H(1)$ is the set of all elements with two-sided inverses. A one-parameter semigroup in $S$ is a continuous, one-to-one function $\sigma:[0,1] \rightarrow S$ such that $\sigma(0)=1$, and $\sigma(a+b)=\sigma(a) \sigma(b)$ for all $a, b \in[0,1]$ for which $a+b \in[0,1]$.

In this paper we obtain the following result on the existence of oneparameter semigroups.

THEOREM 1. Let $S$ be a compact semigroup with identity, and assume that $H(1)$ is not an open set in $S$. Let there be a neighborhood $V$ of the identity containing no other idempotents. Then $S$ contains a one-parameter semigroup $\sigma$ such that $\sigma(a) \oplus H(1)$ for $0<a \leqq 1$. Moreover, $\sigma(a)=\sigma(b) g, g \in H(1)$, implies $a=b$ and $g=1$.

This is a generalization of a previous result of the authors [4, Theorem A] in which it is assumed that $H(1)$ is a Lie group. Our proof will actually establish the following more general result.

THEOREM 2. The conclusion of the previous theorem is correct if $S$ is merely assumed to be locally compact, provided $H(1)$ contains a compact subgroup $G$ open in $H(1)$ but not open in $S$, and provided there is a neighborhood $V$ of $G$ containing no idempotents other than the identity.

In the last part of the paper it is shown that if $S$ is a compact semigroup with zero and identity and no other idempotents, and if $H(1)$ is not an open set in $S$, then $S$ is arcwise connected and $S$ contains a subsemigroup that is an arc with 0 and 1 as its endpoints. Examples are given to show that this result need not be true if there are additional idempotents. Finally, a theorem is stated giving conditions when a semigroup can be locally imbedded in a Lie group.

2. Preliminaries. The topological closure of a set $A$ will be denoted by $A^{-}$. The set-theoretic difference $A$ minus $B$ will be denoted by $A \backslash B$. For the basic properties of topological semigroups see the survey by A. D. Wallace [5].

Let $V$ be the neighborhood of the identity mentioned in the hypotheses of Theorems 1 and 2; without loss of generality we may assume that $V$ has

Presented to the Society, November 30, 1957; received by the editors August 17, 1959.

(1) This work was supported in part by Tulane University's National Science Foundation contract. 
compact closure and that $V^{-}$contains no idempotents other than the identity. Let $N$ denote the index set of a fundamental system of neighborhoods of the identity, partially ordered by inclusion. $N$ is thus a directed set and we give it the discrete topology. Let $\left\{x_{\alpha}\right\}(\alpha \in N)$ denote a net in $V^{-}$(for the properties of nets and subnets see [2, Chapter 2]).

Let $N^{*}$ denote the Stone-Cech compactification of $N$. Any continuous function $f$ from $N$ to a compact space $X$ admits a unique extension to a continuous function $f^{*}: N^{*} \rightarrow X$. In particular, define $f: N \rightarrow V^{-}$by $f(\alpha)=x_{\alpha}$. Choose a point $b \in N^{*} \backslash N$ such that $b$ is a limit point of the directed set $N$, and hold this point $b$ fixed throughout the discussion. A generalized limit is then defined by

$$
\operatorname{Lim} x_{\alpha}=f^{*}(b) .
$$

If the ordinary limit exists it will be denoted by $\lim x_{\alpha}$. If $\left\{y_{\alpha}\right\}$ is another set in $V$-indexed by $N$ we define $\operatorname{Lim} y_{\alpha}$ in a similar manner, using the same point $b$. The generalized limit has the following properties (Gleason [1]).

(i) $\operatorname{Lim} x_{\alpha}$ exists for every set in $V^{-}$indexed by $N$.

(ii) $\operatorname{Lim} x_{\alpha}=\lim x_{\alpha}$ whenever $\lim x_{\alpha}$ exists.

(iii) $\operatorname{Lim} x_{\alpha} y_{\alpha}=\left(\operatorname{Lim} x_{\alpha}\right)\left(\operatorname{Lim} y_{\alpha}\right)$.

(iv) If $x_{\alpha} \in C$, then $\operatorname{Lim} x_{\alpha} \in C^{-}$.

A local semigroup is a Hausdorff space $R$ with a distinguished element 1, called the identity, a neighborhood $V$ of 1 , and a continuous function $m: V \times V \rightarrow R$ which is associative whenever the triple products are defined, and which has the property that $m(x, 1)=m(1, x)=x$. We shall write $x y$ instead of $m(x, y)$.

Let $G$ denote the set of all elements of $V$ having inverses in $V$, in other words, $x \in G$ if and only if $x \in V$ and there is an element $y \in V$ such that $x y=y x=1$. $R$ is said to be an adequate local semigroup if $G$ is a group (not merely a local group). We shall need the following result (see $[4$, Theorem A]).

Let $R$ be a compact adequate local semigroup whose maximal subgroup $G$ is a Lie group. If $G$ is not an open set in $R$, and if there exists a neighborhood of the identity containing no other idempotents, then there exists a one-parameter semigroup $\sigma$ such that $\sigma(a) \notin G$ for $a>0$. Moreover, if $\sigma(a)=\sigma(b) g$ for some $g \in G$, then $a=b$ and $g=1$.

(The last sentence was not included in the statement of Theorem A but may be found in $\$ 4.4 .4$ of $[4]$.)

3. Proof of Theorem 1. Throughout this section we assume the hypotheses of the theorem. As remarked in $\S 2$, we may assume that $V$ is open and has compact closure, and that $V^{-}$contains no idempotents other than 1 . Let $x_{\alpha} \rightarrow 1\left(x_{\alpha} \in \bigvee \backslash H(1), \alpha \in N\right)$.

Lemma 1. For each $\alpha \in N$ there is a positive integer $n(\alpha)$ such that 


$$
\begin{aligned}
x_{\alpha}^{k} & \in V, \quad(1 \leqq k \leqq n(\alpha)), \\
x_{\alpha}^{n(\alpha)+1} & \notin V .
\end{aligned}
$$

Proof. Let $x \in V \backslash H(1)$ and assume that $x^{n} \in V(n=1,2, \cdots)$. Then $\Gamma(x)=\left\{x, x^{2}, x^{3}, \cdots\right\}-\subset V^{-}$. But $\Gamma(x)$ is a compact semigroup and hence contains an idempotent. This can only be 1 , and therefore $\Gamma(x)$ is a group and $x \in H(1)$, which is a contradiction. (See [3] and [5] for the properties of $\Gamma(x)$.)

Let $\tau:[0,1] \rightarrow V^{-}$be defined by

$$
\tau(a)=\operatorname{Lim} x_{\alpha}^{[a n(\alpha)]}
$$

where $[s]$ denotes the greatest integer less than or equal to $s .\left(x^{0}\right.$ is defined to be 1 for all $x \in V$.)

Lemma 2. The function $\tau$ has the following properties.

(i) $\tau(0)=1$,

(ii) $\tau(1) \in V^{-} \backslash V$,

(iii) $\tau(a+b)=\tau(a) \tau(b)$ for $a, b, a+b \in[0,1]$.

Proof. (i) is obvious.

(ii) Since $x_{\alpha} \rightarrow 1$ we have $\tau(1)=\operatorname{Lim} x^{[n(\alpha)]}=\operatorname{Lim} x^{[n(\alpha)]+1}$; the result now follows from (1).

(iii) Fix $a$ and $b$ and let $c(\alpha)=[a n(\alpha)+b n(\alpha)]$. Then $c(\alpha)=[a n(\alpha)]$ $+[b n(\alpha)]+\epsilon(\alpha)$, where $\epsilon(\alpha)=0$ or 1 . Thus $\tau(a+b)=\operatorname{Lim} x_{\alpha}^{c(\alpha)}$ $=\operatorname{Lim} x_{\alpha}{ }^{[a n(\alpha)]} \operatorname{Lim} x_{\alpha}{ }^{[b n(\alpha)]} \operatorname{Lim} x_{\alpha}{ }^{e(\alpha)}$ from which the result follows.

The function $\tau$ is not necessarily continuous. However, in the local semigroup $R^{*}=\{\tau([0,1])\}$ - we shall be able to construct a one-parameter semigroup. Notice that $R^{*}$ is abelian and that $R^{*} \subset V^{-}$(and therefore $R^{*}$ contains no idempotents except the identity).

Lemma 3. Let $Q=\bigcap_{a>0}\{\tau([0, a])\}-$. Then $Q$ is a compact abelian subgroup of $H(1)$.

Proof. By (iii) of Lemma $2, Q^{2} \subset Q$, so $Q$ is a compact abelian semigroup. Furthermore, $1 \in Q$ and $Q$ contains no other idempotents (since $Q \subset V^{-}$). Thus $Q$ is a group, and therefore $Q \subset H(1)$.

Lemma 4. If $a_{n} \rightarrow a\left(a_{n}, a \in[0,1]\right)$, then all cluster points of $\left\{\tau\left(a_{n}\right)\right\}$ are contained in the set $\tau(a) Q$.

Proof. We may assume $a_{n}>a$ for all $n$, or $a_{n}<a$ for all $n$. In the first case, $\tau\left(a_{n}\right)=\tau\left(a_{n}-a\right) \tau(a)$ and the result follows since $a_{n}-a \rightarrow 0$ and therefore $\tau\left(a_{n}-a\right)$ clusters in $Q$. In the second case $\tau(a)=\tau\left(a_{n}\right) \tau\left(a-a_{n}\right)$. Let $x$ be a cluster point of $\left\{\tau\left(a_{n}\right)\right\}$. By taking subnets we may assume that $\tau\left(a_{n}\right) \rightarrow x$, and that $\tau\left(a-a_{n}\right)$ is convergent, say to an element $y$ (necessarily in $Q$ ). Then $\tau(a)=x y$ or $x=\tau(a) y^{-1}$. 
Lemma 5. If $a>0(a \in[0,1])$, then $\tau(a) \notin H(1)$.

Proof. Assume the statement were false, so $\tau(a)=g \in H(1)$ for some $a>0$. Let $x=\tau(a / 2)$, so that $x^{2}=g$. Let $y=x g^{-1}, z=g^{-1} x$. Then $x y=1, z x=1$ and so $x$ has both a left and a right inverse and therefore $x \in H(1)$. Similarly, $\tau\left(a / 2^{n}\right) \in H(1)(n=0,1,2, \cdots)$.

Choose dyadic rationals $r_{n}$ such that $r_{n} a \rightarrow 1^{-}$. Then by Lemma 4, $\tau\left(r_{n} a\right)$ clusters in $\tau(1) Q$. But since $\tau\left(r_{n} a\right) \in H(1)$ for all $n$ this implies that $\tau(1) \in H(1)$, contrary to Lemma 2 (ii).

Lemma 6. There exists $a_{0}>0$ such that $\tau$ is one-to-one on $\left[0, a_{0}\right]$. Moreover, $\tau(a)=\tau(b) g$ with $a \leqq a_{0}$ and $g \in Q$ implies $a=b$.

Proof. Let $c_{0}=\inf \{c \in[0,1]: \tau(c)=\tau(b) g$ for some $b>c, g \in Q\}$. It will be sufficient to prove that $c_{0}>0$, for then $a_{0}$ can be taken to be any positive number smaller than $c_{0}$.

Assume $c_{0}=0$. Then there exist $c_{n}<b_{n}, c_{n} \rightarrow 0, g_{n} \in Q$ such that $\tau\left(c_{n}\right)$ $=\tau\left(b_{n}\right) g_{n}$. Let $d_{n}=b_{n}-c_{n}$. Then

$$
\tau\left(c_{n}\right)=\tau\left(c_{n}\right) \tau\left(d_{n}\right) g_{n}=\tau\left(c_{n}\right) \tau\left(d_{n}\right)^{m} g_{n}^{m}=\tau\left(c_{n}\right) \tau\left(m d_{n}\right) g_{n}^{m}
$$

for every positive integer $m$ such that $m d_{n} \leqq 1$. There exist integers $m(n)$ such that $m(n) d_{n}$ converges to a number $d, 1 / 2 \leqq d \leqq 1$. By taking subnets we may assume

$$
\begin{aligned}
\tau\left(c_{n}\right) & \rightarrow h \in Q, \\
\tau\left(m(n) d_{n}\right) & \rightarrow z \in \tau(d) Q(\text { Lemma } 4), \\
g_{n}^{m(n)} & \rightarrow g \in Q .
\end{aligned}
$$

From (2) we have $h=h z g$ or $z \in Q$, which is a contradiction to Lemma 5 .

Let $R=\tau\left(\left[0, a_{0}\right]\right) Q . R$ is a closed set by Lemma 4 , and therefore $R$ is a compact abelian adequate local semigroup. Also by Lemma $4, Q$ is not an open set in $R$. If $H$ is a closed subgroup of $Q$ then the factor semigroup $R / H$ is an adequate local semigroup whose maximal subgroup is $Q / H$.

Consider the family $F$ of all pairs $(H, \sigma)$ where $H$ is a closed subgroup of $Q$ and $\sigma$ is a one-parameter semigroup in $R / H, \sigma:\left[0, a_{0}\right] \rightarrow R / H$, such that

$$
\sigma(t) \in \pi(\tau(t) Q), \quad\left(0 \leqq t \leqq a_{0}\right),
$$

and

$$
\sigma(a)=\sigma(b) g \quad(g \in Q / H) \text { implies } a=b \text { and } g=1 .
$$

Here $\pi$ denotes the natural projection homomorphism from $R$ to $R / H$. We shall consider all one-parameter semigroups to be defined on the interval $\left[0, a_{0}\right]$. This is no restriction since we may always change parameters and pass to the interval $[0,1]$. Notice that condition (3) implies that 


$$
\sigma(t) \notin Q / H,
$$

The family $F$ is not empty since we may take $H=Q$ in which case $R / Q$ is just the arc $\left[0, a_{0}\right]$, in other words, $R / Q$ is itself a one-parameter semigroup.

We introduce a partial order into $F$ by saying that $(H, \sigma) \leqq\left(H_{1}, \sigma_{1}\right)$ if

(a) $H_{1} \subset H$,

(b) $p \sigma_{1}(t)=\sigma(t),\left(0 \leqq t \leqq a_{0}\right)$,

where $p$ is the natural projection from $R / H_{1}$ to $R / H$. Notice that $p^{-1}(1)$ is isomorphic to $H / H_{1}$. (The symbol 1 will be used indiscriminately to denote the identity of any semigroup with which we are dealing.)

Let $\left(H_{\alpha}, \sigma_{\alpha}\right)$ be a linearly ordered subfamily, and let $K=\bigcap_{\alpha} H_{\alpha}$. One sees easily that there is a one-parameter semigroup $\sigma$ in $R / K$ such that $\left(H_{\alpha}, \sigma_{\alpha}\right)$ $\leqq(K, \sigma)$ for all $\left(H_{\alpha}, \sigma_{\alpha}\right)$ in the subfamily. Indeed, let $R_{\alpha}=R / H_{\alpha}$. Then $R / K$ is the inverse limit of the $R_{\alpha}$, and $\sigma$ may be taken to be the inverse limit of the $\sigma_{\alpha}(t)$. It is easily verified that (3) and (4) hold for $\sigma$. Now let $\left(H_{\alpha}, \sigma_{\alpha}\right)$ be a maximal linearly ordered subfamily. The proof of Theorem 1 will be complete if we can show that in this case $K=\{1\}$. This will follow from the following lemma.

Lemma 7. Let $(K, \sigma) \in F$. Let $K_{1} \subset K$ be a closed subgroup such that $K / K_{1}$ is a Lie group. Then there is a one-parameter semigroup $\sigma_{1}$ in $R / K_{1}$ such that $(K, \sigma) \leqq\left(K_{1}, \sigma_{1}\right)$.

Proof. Let $p$ denote the natural projection from $R / K_{1}$ to $R / K$. Let $R^{\prime}=p^{-1}\left(\sigma\left(\left[0, a_{0}\right]\right)\right)$. Then $R^{\prime}$ is an adequate local semigroup contained in $R / K_{1}$, and the maximal group of $R^{\prime}$ is isomorphic to $K / K_{1}$. We may apply the result stated at the end of $\$ 2$ to conclude that there is a one-parameter semigroup $\gamma:[0,1] \rightarrow R^{\prime}$ such that if $\gamma(a)=\gamma(b) g\left(g \in p^{-1}(1)\right)$, then $a=b$ and $g=1$. Then $\gamma(1) \in \pi_{1}\left(\tau\left(a_{1}\right) Q\right)$ for some $a_{1}\left(0<a_{1} \leqq a_{0}\right)$, where $\pi_{1}$ denotes the natural projection from $R$ to $R / K_{1}$.

Define $\sigma_{1}(t):\left[0, a_{0}\right] \rightarrow R^{\prime}$ by

$$
\begin{aligned}
\sigma_{1}(t) & =\gamma\left(t / a_{1}\right), & \left(0 \leqq t \leqq a_{1}\right), \\
\sigma_{1}(t) & =\left(\sigma_{1}\left(a_{1}\right)\right)^{k} \sigma_{1}(r), & \left(t \leqq a_{0}, t=k a_{1}+r\right) .
\end{aligned}
$$

Then $p \sigma_{1}\left(a_{1}\right)=\sigma\left(a_{1}\right)$. From this it follows easily that $p \sigma_{1}(t)=\sigma(t)\left(0 \leqq t \leqq a_{0}\right)$.

It only remains to show that $\sigma_{1}$ satisfies (4). Assume that $\sigma_{1}(a)=\sigma_{1}(b) g$ for some $g \in Q / K_{1}$. Applying $p$ we have $\sigma(a)=\sigma(b) p(g)$. But (4) holds for $\sigma$ and therefore $a=b$ and $p(g)=1$. This means that $g \in p^{-1}(1)$, but $\gamma$ was so chosen that this implies $g=1$. This completes the proof.

4. Applications. An $(I)$-semigroup is a semigroup on an arc such that one endpoint is a zero and the other is an identity. The general structure is given in $[4$, Theorem B]. In particular, if an $(I)$-semigroup has no idempotents except the two endpoints, then it is isomorphic to one of the following:

(1) $[0,1]$ with the usual multiplication of real numbers, 
(2) $[1 / 2,1]$ with the product $x \cdot y$ defined by $x \cdot y=\max (1 / 2, x y)$, where $x y$ denotes the ordinary multiplication of real numbers.

THEOREM 3. Let $S$ be a compact semigroup with zero and identity and no other idempotents, and assume that $H(1)$ is not an open set. Then $S$ contains an (I)-subsemigroup $J$, and $S$ is arcwise connected.

For the proof of this theorem we shall need the following lemma.

LEMMA 8. Let $S$ be a compact semigroup with zero and identity and no other idempotents. Let $y \in H(1)$. Then $y^{n} \rightarrow 0$, and if $y z=y$ for any $z \notin H(1)$ then $y=0$.

Proof. Let $\Gamma(y)=\left\{y, y^{2}, y^{3}, \cdots\right\}-$. This is a compact semigroup and therefore contains an idempotent. As in the proof of Lemma 1, this idempotent cannot be the identity. But it is known [3, Theorem 1] that if $0 \in \Gamma(y)$ then $y^{n} \rightarrow 0$.

If $y z=y$, then $y z^{2}=y z=y$, and so $y z^{n}=y$. But $z^{n} \rightarrow 0$ and so $y=0$.

We do not know whether $y z=y$ is possible for some $z \neq 1$ in $H(1)$.

Proof of Theorem 3. Let $\sigma$ be the one-parameter semigroup of Theorem 1. Define $\sigma(t)$ for $t \in[1,2]$ by

$$
\sigma(t)=\sigma(1) \sigma(t-1),
$$

It is easy to see that if $a, b, a+b \in[0,2]$ then $\sigma(a+b)=\sigma(a) \sigma(b)$, and that $\sigma(t) \notin H(1)$ for $t>0$.

Assume that $\sigma(a)=\sigma(b) \quad(a<b)$. Then $\sigma(a)=\sigma(a) \sigma(b-a)$, and so by Lemma $8 \sigma(a)=0$. Thus either $\sigma$ is one-to-one on $[0,2]$, or $\sigma(a)=0$ for some $a$.

We may successively extend $\sigma$ into the intervals $[2,3],[3,4]$, etc., so that $\sigma$ is defined for $0 \leqq t<\infty$. By Lemma $8, \lim \sigma(t)=0(t \rightarrow \infty)$. Thus, either $\sigma$ is one-to-one on $[0, \infty]$, or there is a finite number $a$ such that $\sigma(a)=0$ and then $\sigma(t)=0$ for all $t \geqq a$. If we let $a^{\prime}$ be the smallest such $a$, then $\sigma$ is one-to-one on $\left[0, a^{\prime}\right]$. In any case, $J=\sigma([0, \infty])$ is the required $(I)$-semigroup.

To show that $S$ is arcwise connected we observe that if $x \neq 0$ then $x J$ is an arc from $x$ to 0 .

We conjecture that $J$ is a homomorphic retract of $S$. This would imply that $S$ has a "character" (a homomorphism onto an (I)-semigroup).

If $S$ contains an additional idempotent the result need not be true, even when $S$ is connected. For example, let $S$ be the points of the form $(1+1 /(1+x)) \exp (2 \pi i x)(0 \leqq x<\infty)$ together with the unit disc in the complex plane. We multiply as follows:

$$
\begin{aligned}
\left(1+\frac{1}{1+x}\right) e^{2 \pi i x} \cdot\left(1+\frac{1}{1+y}\right) e^{2 \pi i y} & =\left(1+\frac{1}{1+x+y}\right) e^{2 \pi i(x+y)}, \\
\rho_{1} e^{2 \pi i x} \cdot \rho_{2} e^{2 \pi i y} & =\rho_{1} \rho_{2} e^{2 \pi i(x+y)}, \quad\left(\rho_{1}, \rho_{2} \leqq 1\right), \\
\left(1+\frac{1}{1+x}\right) e^{2 \pi i x} \cdot \rho e^{2 \pi i y} & =\rho e^{2 \pi i(x+y)},
\end{aligned}
$$


and take multiplication to be commutative. This is a ray winding asymptotically about the unit disc. The identity $\left(2 e^{2 \pi i 0}=2\right)$ of the ray is an identity for $S$, while the zero of the unit disc is a zero for $S$. In this case there is no (I)-semigroup, in fact no arc at all, from zero to the identity, although a oneparameter semigroup does exist.

We now give another example to illustrate the possibilities when there are additional idempotents. In this example $S$ is the Cantor set endowed with a multiplication which will be described presently. The endpoint 0 will be a zero, 1 will be an identity, and every neighborhood of the identity will contain additional idempotents.

Let $x \in S$. Then $x$ admits a triadic expansion in which only the digits 0 and 2 are used:

$$
x=\sum_{1}^{\infty} \epsilon_{n} / 3^{n}, \quad\left(\epsilon_{n}=0 \text { or } 2\right) .
$$

Let the indices in which two occurs be $n_{1}<n_{2}<\cdots$, and let $J^{*}$ $=\{1,2,3, \cdots, \infty\}$. Define a function $f_{x}: J^{*} \rightarrow J^{*}$ by:

$$
\begin{aligned}
f_{x}(j) & =n_{j}, \\
f_{x}(\infty) & =\infty .
\end{aligned}
$$

This definition is not complete if the expansion (7) has only a finite number of two's. In this case the indices for which a two occurs have a greatest member, $n_{N}$. We complete the definition of $f_{x}$ by

$$
f_{x}(j)=\infty, \quad(j>N) .
$$

In any case the function $f_{x}$ has the following property:

(P) $f_{x}$ is strictly increasing as long as it is finite-valued; once it takes the value $\infty$ it is constantly equal to $\infty$ thereafter.

Conversely, let $g: J^{*} \rightarrow J^{*}$ satisfy $(\mathrm{P})$, and let $x=\sum 2 / 3^{o(j)}$. Then $x \in S$ and $g=f_{x}$. So there is a one-to-one correspondence between the Cantor set $S$ and the set of functions satisfying $(\mathrm{P})$. But the functions form a semigroup under composition, and we take this as the multiplication in $S$ (continuity is easily checked).

The idempotents are precisely the elements 1 and $e_{n}=2 / 3+2 / 3^{2}+\cdots$ $+2 / 3^{n}$; they converge to 1 . The multiplication is order-preserving on one side only: $x<y$ implies $c x \leqq c y$ for all $c \in S$.

Another corollary to Theorems 1 and 2 is the following result (whose proof we omit).

THEOREM 4. Let $S$ be a locally compact semigroup with identity and assume $S$ is an n-dimensional manifold with regular boundary in some neighborhood of 1 . If the hypotheses of Theorem 2 are satisfied and if $\operatorname{dim} H(1)=n-1$, then $S$ can be locally imbedded in a Lie group. 
This result need not be true if $\operatorname{dim} H(1)<n-1$, as the following example shows.

Let $I_{1}$ be $[0,1]$ with ordinary multiplication, and let $I_{2}=[0,1]$ with the multiplication $x \cdot y=\min (x, y)$. Let $S=\left\{(x, y): x \in I_{1}, y \in I_{2}, y \geqq x\right\}$ and give $S$ the coordinatewise multiplication. Then $S$ satisfies the hypotheses of Theorem 4 except that $\operatorname{dim} H(1)=0$. However, every neighborhood of 1 contains elements for which the cancellation law fails, and so no neighborhood of the identity can be imbedded in a group.

5. Concluding remarks. The hypotheses of Theorem 1 are not necessary and sufficient for the existence of a one-parameter semigroup. Let us agree to say that an element $x$ is Archimedean with respect to a neighborhood $V$ of the identity $(x \in V)$ if some power of $x$ is not in $V$. We originally conjectured that if $S$ is a compact semigroup with identity then a necessary and sufficient condition for the existence of a one-parameter semigroup (with $\sigma(t) \notin H(1)$ for $t>0)$ is the existence of a neighborhood $V$ of 1 and a net $x_{a}$ in $V, x_{a} \rightarrow 1$, such that each element $x_{a}$ is Archimedean with respect to $V$. The main difficulty in proving this seems to be in showing that $Q$ is a group (Lemma 3). A. M. Gleason has given us an example of a semigroup with an Archimedean collection for which $Q$ is not a group. Although this example does not necessarily kill the conjecture, it certainly throws doubt on it.

We further conjecture that if $S$ is a locally compact connected semigroup with identity, and if there is a neighborhood of 1 containing no other idempotents, then $S$ contains a one-parameter semigroup.

Finally, we note that R. J. Koch [6] has an unpublished result showing that every compact connected semigroup with identity contains a generalized arc, but an example of R. P. Hunter shows that there need not be any arc having the identity as one endpoint. These results will appear soon.

\section{REFERENCES} 212.

1. A. M. Gleason, Groups without small subgroups, Ann. of Math. vol. 56 (1952) pp. 193-

2. J. L. Kelley, General topology, New York, Van Nostrand, 1955.

3. R. J. Koch, On monothetic semigroups, Proc. Amer. Math. Soc. vol. 8 (1957) pp. 397401.

4. P. S. Mostert and A. L. Shields, On the structure of semigroups on a compact manifold with boundary, Ann. of Math. vol. 65 (1957) pp. 117-143.

5. A. D. Wallace, The structure of topological semigroups, Bull. Amer. Math. Soc. vol. 61 (1955) pp. 95-112.

6. R. J. Koch, Arcs in partially ordered spaces, Pacific J. Math. vol. 9 (1959) pp. 723-728.

Tulane University, New Orleans, Louistana

UNIVERSITY OF MICHIGAN, ANn Arbor, Michigan 